\title{
Idarubicin and cytarabine in combination with gemtuzumab ozogamicin (IAGO) for untreated patients with high-risk MDS or AML evolved from MDS: a phase II study from the EORTC and GIMEMA Leukemia Groups (protocol 06013)
}

\author{
Theo de Witte ${ }^{1,8} \cdot$ Stefan Suciu $^{2} \cdot$ Liv Meert ${ }^{2} \cdot$ Constantijn Halkes $^{3} \cdot$ Dominik Selleslag $^{4}$. \\ Dominique Bron $^{5} \cdot$ Sergio Amadori ${ }^{6} \cdot$ Roel Willemze $^{3} \cdot$ Petra Muus $^{1} \cdot$ Frédéric Baron $^{7}$
}

Received: 20 May 2015 / Accepted: 17 August 2015 / Published online: 26 September 2015

(C) The Author(s) 2015. This article is published with open access at Springerlink.com

\begin{abstract}
The primary objective of this trial was to assess the feasibility, toxicity profile, and antitumor activity of gemtuzumab ozogamicin (GO) combined with a chemotherapy remission-induction regimen in adults with untreated highrisk myelodysplastic syndrome (HR-MDS) or secondary acute myeloid leukemia (sAML). In this phase II trial, 30 patients with median age of 58 years received 1 day of GO as a 1-h infusion at the dose level of $5 \mathrm{mg} / \mathrm{m}^{2}$ on day 7 of the remission-induction course further consisting of a continuous infusion of cytarabine $100 \mathrm{mg} / \mathrm{m}^{2} /$ day for 10 days and idarubicin $12 \mathrm{mg} / \mathrm{m}^{2} /$ day on days 1,3 , and 5 . A consolidation course, consisting of intermediate-dose cytarabine (A) and idarubicin (I) followed by hematopoietic stem cell transplantation (HSCT) was planned for patients in complete remission (CR). The primary endpoints were response rate (CR/CRi) and severe toxicity rate. The secondary endpoint(s) were survival and progression-free survival (PFS) from start of treatment. Thirteen patients ( $43 \%$ ) achieved CR (eight patients) or
\end{abstract}

Theo de Witte

theo.dewitte@radboudumc.nl

1 Radboud University Medical Centre, Nijmegen, The Netherlands

2 EORTC Headquarters, Brussels, Belgium

3 Leiden University Medical Center, Leiden, The Netherlands

4 St Jan's Hospital, Brugge, Belgium

5 Institut J. Bordet (ULB), Brussels, Belgium

6 Tor Vergata University Hospital, Rome, Italy

7 University of Liege, Liege, Belgium

8 Department of Tumorimmunology, Radboud Institute of Molecular Life Sciences, Radboud University Medical Centre, PO Box 9101, 6500 HB Nijmegen, The Netherlands
CR with incomplete hematopoietic recovery (CRi) (five patients). In patients who achieved $\mathrm{CR}$ or $\mathrm{CRi}$, the median time to recovery of neutrophils to $0.5 \times 10^{9} / 1$ and of platelets to $>50 \times 10^{9} / 1$ was 29 and 30 days, respectively. Grade 3 to 4 severe toxicities occurred in nine patients. The most prominent was liver toxicity, as shown by elevated bilirubin levels in 16 patients and one case of nonfatal veno-occlusive disease (VOD). All 13 patients with $\mathrm{CR} / \mathrm{CRi}$ received consolidation therapy, which was followed by allogeneic HSCT in five patients and autologous HSCT in three patients. According to the statistical design of the study, the idarubicin and cytarabine in combination with gemtuzumab ozogamicin (IAGO) regimen did not show sufficient activity to warrant further exploration of this regimen in adult patients with HR-MDS or sAML.

Keywords High-risk myelodysplastic syndromes . Secondary acute myeloid leukemia $\cdot$ Gemtuzumab ozogamicin · Chronic myelomonocytic leukemia $\cdot$ Liver toxicity $\cdot$ Cytogenetic risk score

\section{Introduction}

AML-like therapy for patients with high-risk myelodysplastic syndrome (MDS) has been accepted less widely than for patients with de novo AML $[1,2]$. The European LeukemiaNet guidelines on MDS recommend that induction chemotherapy should be considered for fit patients without a suitable donor who are younger than age 65 to 70 years and have $10 \%$ or more bone marrow blasts without adverse cytogenetic characteristics [3]. Prognostic factors for outcome after AML-like therapy in MDS have been studied less frequently than in de novo AML [2, 4-6]. Cytogenetic abnormalities, age, antecedent 
hematological disease (AHD), performance status, and applied treatment are prognostic factors for survival and event-free survival (EFS) [7]. The results of intensive chemotherapy in patients with advanced stages of MDS have improved with complete remission $(\mathrm{CR})$ rates now ranging between 44 and $64 \%$ [4, 7-11]. Remission after chemotherapy usually lasts less than 12 months [9, 12]. The higher incidence of adverse cytogenetic characteristics and the higher expression of the multidrug resistance 1 gene (MDR1) in MDS compared to de novo AML may explain the inferior response to chemotherapy [13-16].

The rationale for this study was to improve eradication of the malignant clones in high-risk MDS and secondary AML (sAML). Incomplete eradication of these clones is the main cause of treatment failure demonstrated as a relatively low CR rate and a high early relapse rate of more than $50 \%$, unless treated by allogeneic hematopoietic stem cell transplantation (HSCT). Therefore, new studies should focus on new and better remission-induction and consolidation regimens. For the development of a new prospective, randomized study, it is necessary to perform phase II studies. We decided to test a new reductionremission regimen as primary treatment in the same patient population participating in a previous MDS study [17]. We elected to incorporate gemtuzumab ozogamicin (GO) into regular remission-induction regimen. GO consists of a humanized antiCD33 monoclonal antibody linked to calicheamicin, a potent antitumor antibiotic [18]. GO binds to CD33, an antigen expressed on the surface of $>90 \%$ of AML blast cells. Binding of GO is followed by internalization and toxin release intracellularly leading to DNA damage and cell death [19]. In studies of older patients with AML in first relapse, tolerable toxicity and a response rate of $30 \%$ was reported following two infusions of GO $9 \mathrm{mg} / \mathrm{m}^{2}$, although full platelet recovery did not occur in roughly half of responders [20]. These results led to the drug regulatory approval in the United States for use in older patients in first relapse for whom standard therapy was unsuitable, setting the stage for its evaluation in patients with newly diagnosed high-risk MDS. Our group developed a prospective randomized trial combining two infusions of GO $6 \mathrm{mg} /$ $\mathrm{m}^{2}$ followed by standard remission-induction chemotherapy in fit older patients [21]. This combination provided no benefit compared to the control arm and appeared too (hemato)toxic in patients older than 70 years. Patients with secondary AML younger than 70 years might be an exception [22]. Based on this experience, we selected the GO dosage of $5 \mathrm{mg} / \mathrm{m}^{2}$ as a single infusion on day 7 of the chemotherapy regimen [21, 23].

\section{Patients and methods}

\section{Patients and eligibility}

Thirty-one patients were registered in this study between January 2003 and March 2006. One patient was ineligible.
Therefore, a total number of 30 patients were evaluable. The patients had to meet the following disease criteria: (1) highrisk MDS, defined as refractory anemia with excess of blasts in transformation (RAEBt), RAEB $>10 \%$ BM blasts, other forms of MDS with multiple ( $\geq 3$ ) chromosomal abnormalities or chromosome seven abnormalities and/or severe cytopenias defined as follows: neutrophil count $<0.5 \times 10^{9} / 1$ and/or platelet count $<20 \times 10^{9} /$ l; (2) chronic myelomonocytic leukemia (CMMoL) with $>5 \%$ BM blasts or with $>16 \times 10^{9} / 1$ neutrophils or with $2.6 \times 10^{9} / 1$ monocytes in the blood; (3) secondary AML after overt MDS of more than 6 months duration. In addition, the following criteria were required: age 16 to 70 years and adequate renal and liver function, defined as $1.5 \times$ upper limit of normal (ULN). Patients who had already received chemotherapy and/or radiotherapy were not eligible. All participants gave their informed consent. The study was registered in clinicaltrials.gov (NCT00077116).

\section{Study design}

The cytarabine in combination with gemtuzumab ozogamicin (IAGO) study was a phase II study carried out by the European Organization for Research and Treatment of Cancer (EORTC) Leukemia group and the Gruppo Italiano Malattie Ematologiche dell Ádulto (GIMEMA).

The primary objective of this trial was to assess the feasibility, toxicity profile, and antileukemic/anti-MDS activity of GO in combination with a standard chemotherapy regimen consisting of idarubicin and cytarabine in previously untreated patients with high-risk MDS or sAML developing after a preceding period with MDS during 6 months.

Secondary objectives were to monitor hepatotoxicity, in particular veno-occlusive disease (VOD), to determine the severity of pancytopenia and duration of recovery in patients who reached complete remission or $\mathrm{CR}$ with incomplete hematopoietic recovery (CRi).

Patients who met eligibility criteria had to be prospectively registered at the EORTC Headquarters in Brussels, Belgium.

The remission-induction course consisted of a continuous infusion of cytarabine $100 \mathrm{mg} / \mathrm{m}^{2} /$ day for 10 days in combination with idarubicin $12 \mathrm{mg} / \mathrm{m}^{2} /$ day on days 1,3 , and 5 as 5 min infusions and GO on day 7 as a 1-h infusion at the dose level of $5 \mathrm{mg} / \mathrm{m}^{2}$ (IAGO).

Response assessment was planned around day 31 after the start of the induction course. The revised recommendations of the International Working Group for Diagnosis, Standardization of Response Criteria, Treatment Outcomes, and Reporting Standards for Therapeutic Trials in MDS [24] were used. A CR required normalization of the marrow blasts (less than $5 \%$ ) and recovery of normal hematopoiesis with a neutrophil count of $1 \times 10^{9} / 1$ or more and a platelet count of $100 \times 10^{9} / 1$ or more in addition to disappearance of all clinical, laboratory, or radiologic evidence of disease. CRi had criteria 
Table 1 Patient characteristics and survival rates at 1 and 2 years

\begin{tabular}{|c|c|c|c|}
\hline \multirow[t]{2}{*}{ Patient characteristics } & \multirow[t]{2}{*}{ All patients (\%) } & \multicolumn{2}{|c|}{ Survival (SE) } \\
\hline & & At 1 year & At 2 years \\
\hline All patients $(\mathrm{M} / \mathrm{F})$ & $30(21 / 9)$ & $53(9)$ & $27(8)$ \\
\hline Age $<55$ years & & $44(17)$ & $33(16)$ \\
\hline Age $\geq 55$ years & & $57(11)$ & $24(9)$ \\
\hline \multicolumn{4}{|l|}{ Performance status (WHO, $0-4$ ) } \\
\hline 0 & 17 & - & - \\
\hline $1 / 2$ & 3 & - & - \\
\hline MDS/sAML classification & 21 & $48(11)$ & $24(9)$ \\
\hline RAEB-1 & 1 & - & - \\
\hline RAEB-2 & 12 & - & - \\
\hline CMML $<5 \%$ marrow blasts & 1 & - & - \\
\hline $\mathrm{CMML} \geq 5 \%$ marrow blasts & 2 & - & - \\
\hline RAEBt/sAML & 14 & $64(15)$ & - \\
\hline Hemoglobin $(\mathrm{g} / \mathrm{dl})<10$ & 25 & - & - \\
\hline Hemoglobin $(\mathrm{g} / \mathrm{dl}) \geq 10$ & 5 & - & - \\
\hline All nucleated cells $<1.8 \times 10^{9} / 1$ & 17 & - & - \\
\hline All nucleated cells $\geq 1.8 \times 10^{9} / 1$ & 3 & - & - \\
\hline Platelets $<100 \times 10^{9} / 1$ & 21 & - & - \\
\hline Platelets $\geq 100 \times 10^{9} / 1$ & 9 & - & - \\
\hline \multicolumn{4}{|l|}{ Cytogenetics (IPSS) } \\
\hline Good & 10 & $90(9)$ & $40(15)$ \\
\hline Intermediate & 9 & $56(17)$ & $33(16)$ \\
\hline Poor & 7 & 0 & 0 \\
\hline ND/failure & 4 & $50(25)$ & $25(22)$ \\
\hline \multicolumn{4}{|l|}{ IPSS } \\
\hline Intermediate-1 & 2 & $50(35)$ & $50(35)$ \\
\hline Intermediate- 2 & 6 & $67(19)$ & $33(19)$ \\
\hline Poor & 13 & $39(13)$ & $15(10)$ \\
\hline sAML & 9 & $67(16)$ & $33(16)$ \\
\hline \multicolumn{4}{|c|}{ Interval from diagnosis to start of treatment } \\
\hline$\leq 2$ months & 15 & - & - \\
\hline$>2$ months (range $2-53$ ) & 15 & - & - \\
\hline \multicolumn{4}{|l|}{ CRIANT score (points) } \\
\hline $0-20$ & $2(7)$ & $54(14)$ & $23(12)$ \\
\hline $20-<50$ & $13(43)$ & $47(13)$ & $20(10)$ \\
\hline$\geq 50$ & $15(50)$ & $47(13)$ & $33(16)$ \\
\hline
\end{tabular}

$S E$ standard error, $R A E B$ refractory anemia with excess of blasts, $C M M L$ chronic myelomonocytic leukemia, RAEBt RAEB in transformation, $S A M L$ secondary acute myeloid leukemia, IPSS international prognostic scoring system, CRIANT Score for details, see "Methods" section

similar to CR but with neutrophils between $0.5 \times 10^{9}$ and $1 \times$ $10^{9} / 1$ and/or platelet counts between $50 \times 10^{9}$ and $100 \times 10^{9} / \mathrm{L}$. Partial remission (PR) required blood recovery as for CR but with both a decrease in marrow blasts of at least $50 \%$ and not more than $25 \%$ abnormal cells in the marrow.

In case PR was achieved after the first course, a similar second remission-induction course was given. In case of CR or CRi after one or two induction courses, a single consolidation course was recommended consisting of intermediate-dose cytarabine $\left(500 \mathrm{mg} / \mathrm{m}^{2}\right.$ every $12 \mathrm{~h}$ in a 2 -h infusion, on days $1-6)$ and idarubicin $\left(10 \mathrm{mg} / \mathrm{m}^{2} /\right.$ day as 5 -min infusion, on days $4,5,6)$ to be followed by either an allogeneic HSCT or an autologous HSCT [17].

Reasons to stop treatment in the protocol: normal completion of the protocol after one or two courses IAGO, ineligibility, non-compliance of the patient, excessive toxicity, death, and loss to follow-up (LFU).

\section{Endpoints}

The main endpoints were the best complete response (CR/ $\mathrm{CRi}$ ) rate after one or two courses of IAGO and severe toxicity rate observed during or after the completion of IAGO. The secondary endpoint(s) were the following: survival and progression-free survival (PFS) from start of treatment. Overall survival (OS) was calculated, from the date of start of treatment until date of death (whatever the cause). Patients still alive were censored at the moment of last visit/contact.

PFS was calculated from the start of the first course of IAGO until first date of relapse in patients who reached CR/ $\mathrm{CRi}$ or progression or until death (whatever the cause and whichever occurred first). Patients still alive, in first CR/CRi (i.e., without relapse), have been censored at the moment of last visit/contact.

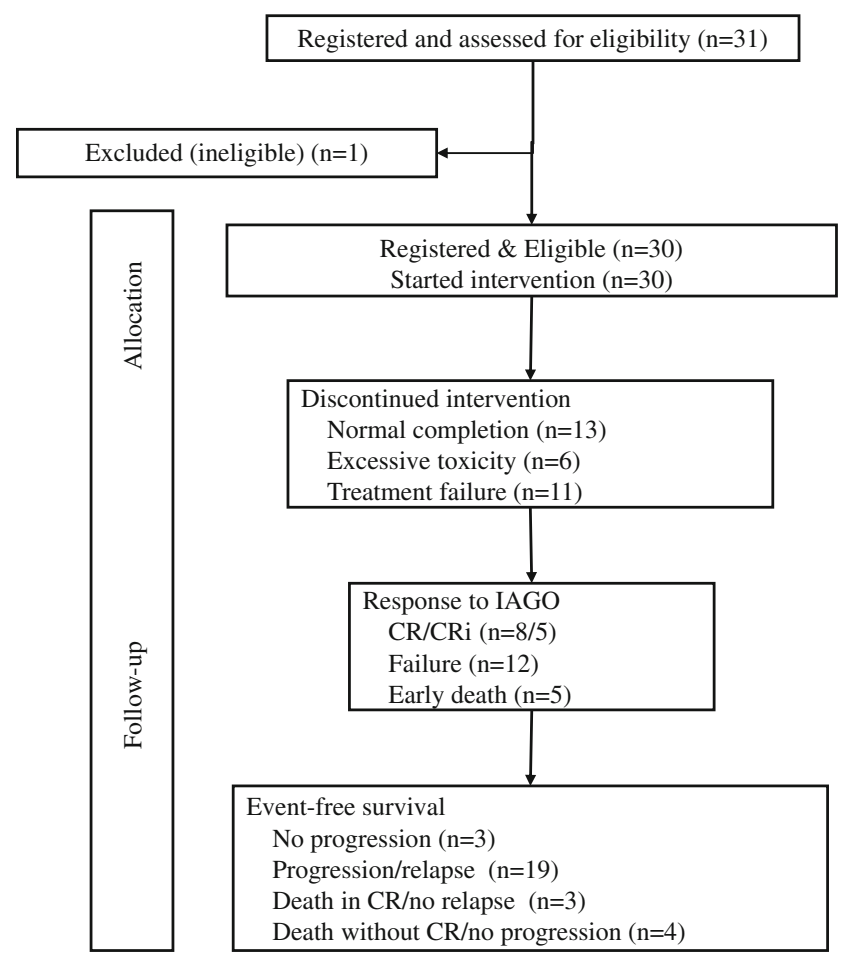

Fig. 1 Treatment plan and flow diagram 
Table 2 Overview of toxicities during IAGO-1 course

\begin{tabular}{lll}
\hline Type of toxicity & All grades & Grades 3-4 \\
\hline All & 30 & \\
Allergy & 3 & - \\
Hypertension & 5 & - \\
Cardiac & 16 & 7 \\
Fatigue & 5 & 2 \\
Skin rash & 15 & 4 \\
Anorexia & 13 & 4 \\
Stomatitis/mucositis & 4 & 2 \\
Hemorrhage & Not applicable & 1 \\
Veno-occlusive disease & 9 & 9 \\
Febrile neutropenia & 16 & 5 \\
Elevated bilirubin & 5 & - \\
Elevated creatinine & 18 & 2 \\
Elevated AST & 16 & 2 \\
Elevated ALT & &
\end{tabular}

Adverse events for each treatment course were recorded according to the National Cancer Institute's Common Terminology Criteria for Adverse Events (version 2.0).

\section{Statistical design and methods}

A Bryant-Day one-step design was used, including response (CR/CRi) and excessive toxicity as the primary endpoint. The following design parameters were considered:

- $\mathrm{P} 0$ is the largest $\mathrm{CR} / \mathrm{CRi}$ probability which, if true, implies that the therapeutic activity does not warrant further investigation of the regimen (IAGO). In the present trial, $\mathrm{P} 0$ has been taken as $50 \%$.

- $\mathrm{P} 1$ is the lowest $\mathrm{CR} / \mathrm{CRi}$ probability which, if true, implies that the therapeutic activity does warrant further investigation of the regimen provided acceptable severe acute toxicity occurrence. In the present trial, P1 has been taken as $75 \%$.
- (1-T0) is the smallest severe toxicity occurrence probability which, if true, implies that the therapeutic toxicity is unacceptable and the regimen does not warrant further investigation. In the present trial, (1-T0) was taken as $50 \%$.

- (1-T1) is the largest severe toxicity occurrence probability which, if true, implies that the therapeutic toxicity is acceptable and the regimen does warrant further investigation provided acceptable response rate. In the present trial, (1-T1) was taken as $20 \%$.

- Beta error: the accepted probability of rejecting from further trials a regimen with a true $\mathrm{CR} / \mathrm{CRi}$ rate at least equal to $\mathrm{P} 1$ and a true toxicity rate equal to or lower than (1-T1). In the present trial, beta was taken as 0.10 .

- Alpha error: the accepted probability of recommending for further investigation a regimen with a true $\mathrm{CR} / \mathrm{CRi}$ rate equal to or lower than P0. It is also the accepted probability of recommending for further trials a regimen with a true severe toxicity rate equal to or higher than (1-T0). In the present trial, the two alphas were taken as 0.10.

Based on these parameters, a total of 28 patients had to be assessed for overall response and toxicity, and the following decision rule had to be applied:

- If $\leq 17(17 / 28=60.7 \%), \mathrm{CR} / \mathrm{CR}$ is were observed, or if $\geq 11(11 / 28=39.3 \%)$, patients had severe toxicities, the conclusion that IAGO is not enough active or is too toxic, and should not be further investigated; otherwise, the conclusion will be that IAGO is active and feasible, and should be further investigated in this patient population.

A total of 31 patients has been finally been entered, in order to cope with the exclusion of ineligible patients or of those who did not start IAGO course. Among them, one patient was considered to be ineligible by the study coordinator because of incorrect diagnosis.

The time to event distributions (PFS and OS) were estimated using the Kaplan-Meier technique, and the standard errors

Table 3 Overview of outcome of nine patients with severe toxicities

\begin{tabular}{lll}
\hline Pt nr & Duration survival (days) & Main complication/reason off protocol \\
\hline 2 & 23 & Febrile neutropenia leading to diffuse intravascular coagulation and to liver and renal failure; VOD not likely \\
6 & 32 & Cardiac arrest \\
9 & 11 & Pulmonary infiltrates; died from pulmonary failure due capillary leakage \\
13 & 1129 & VOD; normal completion of protocol \\
20 & $1254+$ & Bilateral interstitial pneumonitis + normal completion \\
24 & 37 & Lung infection, pulmonary insufficiency, and multi-organ failure \\
25 & $1035+$ & Iatrogenic hematothorax + normal completion \\
26 & 103 & Grade 3 bilirubin elevation and liver lesions; grade 3 infection \\
29 & 36 & Cerebral hemorrhage day 35; persisting thrombocytopenia \\
\hline
\end{tabular}


Table 4 Hematopoietic recovery after first course of IAGO in 13 patients with $\mathrm{CR}$ or CRi

\begin{tabular}{ll}
\hline Recovery from start of IAGO & $\begin{array}{l}\text { Medium number of days } \\
\text { (95\% confidence intervals) }\end{array}$ \\
\hline $\mathrm{PMN}>0.5 \times 10^{9} / 1$ & $30(28$ to 32$)$ \\
$\mathrm{PMN}>1.5 \times 10^{9} / 1$ & $35(31$ to 43$)$ \\
Platelets $>50 \times 10^{9} / 1$ & $29(28$ to NR) \\
Platelets $>100 \times 10^{9} / 1$ & $42(28$ to NR) \\
\hline
\end{tabular}

$P M N$ polymorphic nucleated cells, $N R$ not reached

(SE) of the estimates were obtained via the Greenwood formula. SAS 9.3 software (SAS Institute, Cary, NC) was used for the statistical analyses.

The CRIANT score was used to analyze the outcome according to adapted prognostic criteria [2]. The score was created based on the weight expressed in points of five criteria: cytogenetics (good, 0; intermediate, 20; poor, 40; unknown, 20), $\mathrm{WBC} \times 10^{9} / 1(<25,0 ; \geq 25,20)$, age in years $(\leq 45,0 ; 45-55$, $20 ;>55,22)$, antecedent hematological disorder in months $(\leq 6$, $0 ;>6,13)$, and number of cytopenias $(0-2,0 ; 3,15)$. The CRIANT score distinguished three risk groups: low risk, $<20$ points; intermediate risk, $20-49$ points; and high risk, $\geq 50$ points.

\section{Results}

Patient characteristics are shown in Table 1. The median age was 58 years with a range from 21 to 66 years. Fourteen patients had progressed to RAEBt [5] or secondary AML [9]. All 30 eligible patients started the planned treatment (Fig. 1). All patients received the full dosage of idarubicin, two patients received a slightly modified dosage of cytarabine, and one patient a modified dosage of GO. Three patients received a second course of IAGO after a PR to the first course.

\section{Side effects}

Side effects occurred in 25 patients during and after the first course, including grade 3 to 4 toxicities in nine patients (see Table 2). Besides fatigue, the most prominent toxicity concerned liver toxicity, as shown by elevated bilirubin levels in 16 patients, including grade 3 to 4 in five patients. One case of clinically relevant VOD has been diagnosed during the first course of IAGO. This patient recovered completely, and he could complete his treatment (see Table 3). Two out of the three patients who received the second course developed severe liver toxicity as shown by grade 3-4 elevated bilirubin levels, but these patients did not develop VOD. Five (17\%) patients died within 40 days after start of IAGO-1 due to treatment related toxicities; for details, see Table 3.

\section{Responses and duration of hypoplasia}

Thirteen (43, $90 \%$ CI $(28,60 \%))$ patients achieved a major response, including eight patients with $\mathrm{CR}$ and five patients with CRi (Fig. 1). Among the latter group, one patient achieved PR after one course and entered CRi after two
Table 5 Post-remission therapy and outcome

\begin{tabular}{|c|c|c|c|c|c|}
\hline Pt nr & Response & Type of SCT & $\mathrm{SCT}^{\mathrm{a}}$ & Relapse $^{\mathrm{a}}$ & Survival $^{\mathrm{a}}$ : cause of death ${ }^{\mathrm{b}}$ \\
\hline 4 & $\mathrm{CR}$ & Auto & 120 & 209 & 269 \\
\hline 10 & $\mathrm{CR}$ & No & & 336 & 490 \\
\hline 11 & $\mathrm{CR}$ & Auto & 194 & No & 203: infection \\
\hline 13 & $\mathrm{CRi}$ & No & & 414 & 1129 \\
\hline 15 & CRi & No & & No & $1463^{\mathrm{c}}: \mathrm{CR}$ \\
\hline 16 & $\mathrm{CR}$ & Allo & 173 & No & $1420^{\mathrm{c}}: \mathrm{CR}$ \\
\hline 17 & $\mathrm{CR}$ & Auto & 127 & 132 & 160 \\
\hline 19 & $\mathrm{CR}$ & Allo & 205 & No & 441: GVHD \\
\hline 20 & CRi & Allo & 576 & 471 & $1254^{\mathrm{c}}$ : second CR \\
\hline 22 & $\mathrm{CR}$ & No & & 305 & 405 \\
\hline 23 & $\mathrm{CR}$ & Allo & 160 & No & 168: NRM \\
\hline 25 & CRi & No & & No & $1035^{\mathrm{c}}: \mathrm{CR}$ \\
\hline 28 & CRi & Allo & 103 & No & $1042^{\mathrm{c}}: \mathrm{CR}$ \\
\hline 31 & Failure & Allo & 161 & Progression & $827^{\mathrm{c}}:$ active disease \\
\hline
\end{tabular}

$C R$ complete remission, $C R i \mathrm{CR}$ with incomplete recovery of platelets and leukocytes

\footnotetext{
${ }^{a}$ Number of days after starting treatment

${ }^{\mathrm{b}}$ Active disease, if not mentioned specifically

${ }^{\mathrm{c}}$ Patient still alive at last follow-up
} 
courses of the IAGO schedule. CR or CRi only occurred in patients with good and intermediate cytogenetic risk score according to IPSS (six out of 10 and five out nine, respectively), while none of the seven patients with poor-risk cytogenetic characteristics achieved CR in this study. The hematopoietic recovery of patients who achieved $\mathrm{CR}$ or CRi was clearly delayed as shown by the median recovery of neutrophils to $0.5 \times 10^{9} / 1$ of 30 days and of the platelets to $>50 \times 10^{9} / 1$ of 29 days. For further details, see Table 4 . Thirteen patients (43\%) had a normal completion of the protocol consisting of CR or CRi (Fig. 1). The other patients went off protocol due to toxicity in six patients or due to treatment failure in 11 patients (37\%) (Fig. 1).

\section{Post-remission therapy}

All 13 patients with CR/CRi received consolidation therapy which was followed by allogeneic HSCT in five patients and autologous HSCT in three patients. Another patient who failed to respond to the induction also received an allogeneic transplantation. Five patients are alive without evidence of disease, including one patient ( $\mathrm{nr} 20$ ) in second CR after alloSCT; for
Fig. 2 a Overall survival after treatment with IAGO and postremission therapy. b Progressionfree survival after treatment with IAGO and post-remission therapy

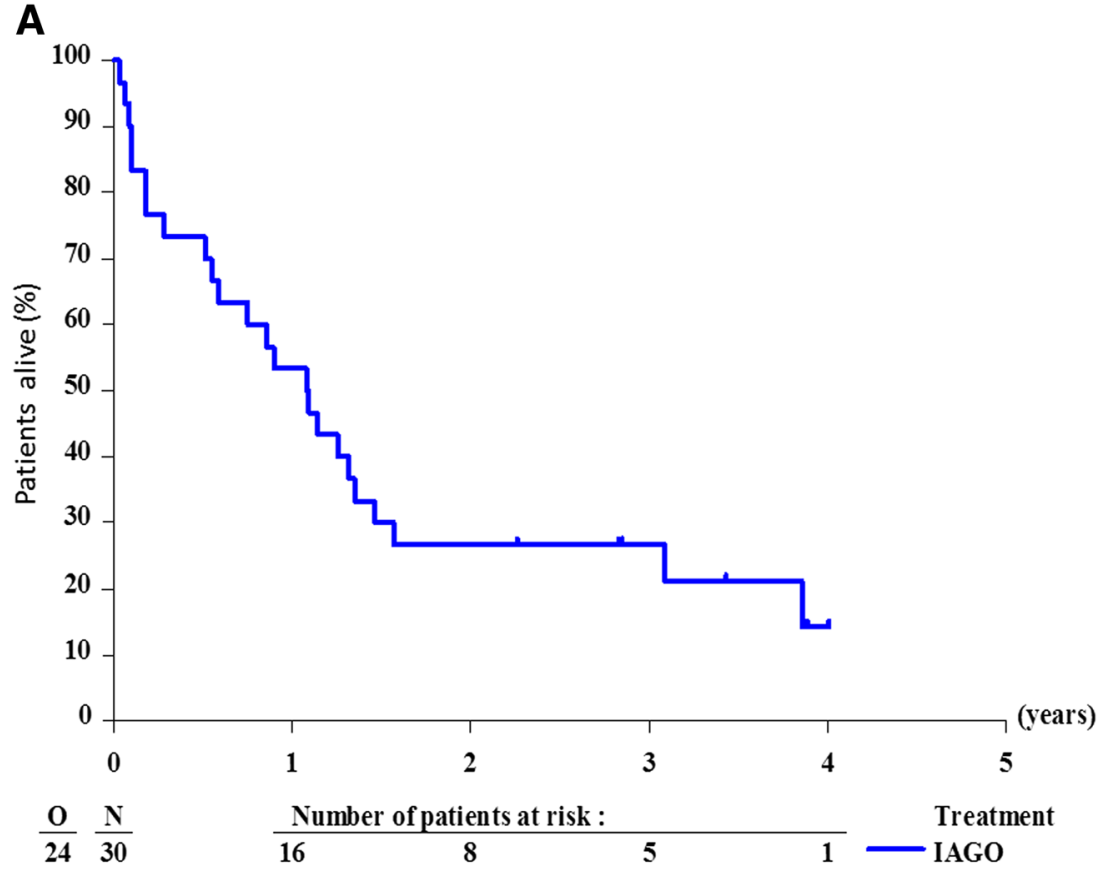

B

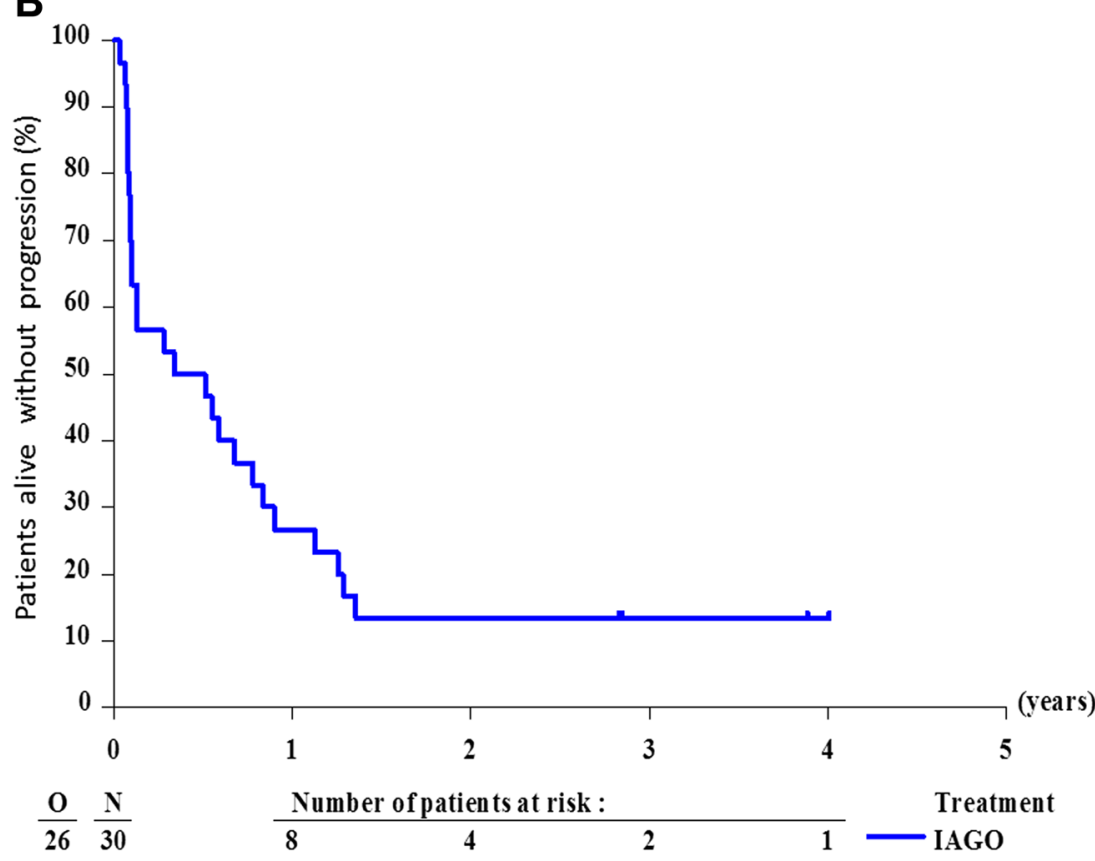


details, see Table 5. Three patients have died in CR due to complications after HSCT: two patients after alloHSCT and one patient after autoHSCT.

\section{Outcome and prognostic factors}

At the time of final evaluation, four patients (13\%) were still alive without signs of progression and two additional patients were alive either with active disease or in second CR (Table 5). Seventeen patients have died with active disease and seven patients due to toxicity, including three patients in CR. The median follow-up was 3.4 years. The median survival was 1.09 years (95\% CI 0.75 to 1.57$)$. The survival rate at 1 year from start of treatment was $53 \%$ (SE 9\%) and at 2 years was $27 \%$ (SE $8 \%$ ) (Table 1, Fig. 2a).

The median PFS was 5.1 months (95\% CI 1.6 to 10.8), and the PFS rate at 1 year was $27 \%$ with SE of $8 \%$ (Fig. 2b).

Age ( $<55$ versus $\geq 55$ years) had no impact on survival (Table 1). None of the seven patients with IPSS poor-risk cytogenetic characteristics was alive at 1 year after start of IAGO, while the 1-year survival in patients with good or intermediate risk cytogenetics was $90.0 \%$ (SE $9 \%$ ) and $56 \%$ (SE $17 \%$ ), respectively; for details and 2-year survival rates, see Table 1 and Fig. 3. The percentage of marrow blasts, subdivided according to less than $20 \%$ or $20 \%$ and more did not influence survival, with a 1-year survival rate of $47 \%$ (SE 11) and $64 \%$ (SE $15 \%$ ), respectively. The prognostic importance of IPSS regarding survival was weak, probably due to the important weight allocated to the percentage of marrow blasts and/or due to the limited number of patients in each IPSS subgroup (Table 1). Fifty percent of the patients were classified as poor-risk according to the recently developed CRIANT risk score [2] and only two patients as goodrisk patients. The survival of the poor-risk and intermediate risk patients was similar (Table 1 ).

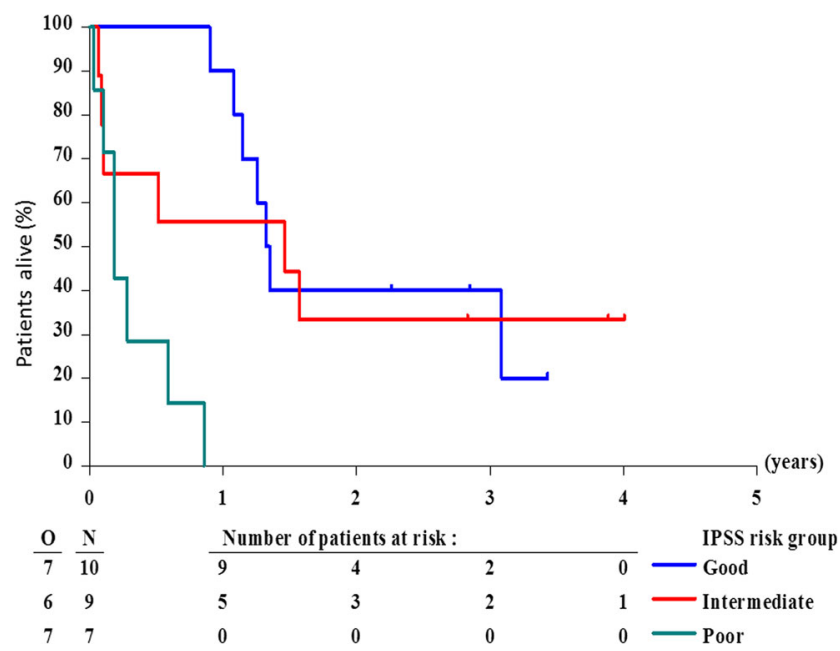

Fig. 3 Overall survival in cytogenetic subgroups according to IPSS

\section{Discussion}

In view of the observed CR/CRi rate of $43 \%$ (13 out of 30 patients) with a $90 \%$ CI of $(27.9,59.8 \%)$, which did not contain the targeted CR/CRi rate of $75 \%$, the Bryant-Day one-step (negative) decision rule of this study was met. This $\mathrm{CR}$ rate is lower than the generally observed remission rates after various intensive chemotherapy regimens [12, 17, 20, 25, 26]. This indicates that the IAGO schedule was not sufficiently active to warrant further exploration in this category of patients.

The major cause of treatment failure in this study was insufficient efficacy of the IAGO schedule. Similar studies, exploring new schedules in high-risk MDS patients, have resulted in higher CR rates, including the $71 \% \mathrm{CR}$ rate in the FLAG schedule (fludarabine, high dose cytarabine, and G-CSF) [26]. Several randomized studies of GO combined with intensive chemotherapy have been performed in patients with elderly primary AML. A meta-analysis of five prospective studies (in total 3325 patients) [27] showed that addition of GO did not increase the proportion of patients entering $\mathrm{CR}$ with an odds ratio (OR) of 0.91 and a $95 \%$ confidence interval (CI) of $0.77-1.07(p=0.3)$. However, the addition of GO significantly improved survival (OR 0.90; $95 \%$ CI 0.82-0.98, $p=0.03$ ), although the 5-year OS difference was only around $3 \%$ : $35.5 \%$ (GO arm) versus $32.2 \%$ (control group). In addition, patients with adverse cytogenetic characteristics did not benefit from the addition of GO in contrast to patients with favorable or intermediate cytogenetic features [27]. The great majority of patients in this meta-analysis were patients with de novo AML. Only two studies [28, 29] included patients with secondary AML and only one study included high-risk MDS patients [29].

Toxicity was an important contributing factor to the general outcome in this study, since six out of the 17 patients went off protocol due to toxicity. Toxicity was fatal within 40 days after starting treatment in five patients $(16.7 \%)$. Overall, the observed grade $3-4$ toxicity was $33.3 \%$, and its $90 \%$ CI $(16.6,46.5 \%)$ did not cover the inacceptable toxicity rate of $50 \%$. In an earlier study of our group [30], we treated a younger age group (median age of 47 years) of 194 patients with the same remission-induction regimen (idarubicin, cytarabine, and etoposide (ICE)) with the exception of etoposide which has been replaced by GO in the present study. The CR rate in that study was $54 \%$ and 29 patients $(16 \%)$ died during the remission-induction phase, which is comparable to the $17 \%$ observed in the current study [30]. The CR rate was $58 \%$ in a more recent study [17] utilizing the same schedule (ICE) as the previous study [30]. The median patient age of the patients in this study was lower (52 versus 58 years), but the percentage of patients with poor-risk cytogenetics was higher in the previous study: 31 versus $23 \%$ in the IAGO study [17]. None of 
the seven patients with poor-risk cytogenetic characteristics entered $\mathrm{CR} / \mathrm{CRi}$, and none of these patients was alive at 1 year after starting treatment. In the previous study, three risk groups were distinguished: a good-risk group with a score $<20$, an intermediate risk group with a score 20 $<50$, and a poor-risk group with a score $\geq 50$. The 5 -year estimated survival rates were $69 \%(\mathrm{SE}=10.2 \%), 37 \%$ $(\mathrm{SE}=5.6 \%)$, and $5 \%(\mathrm{SE}=2.1 \%)$ for the three groups, respectively [2]. Poor-risk cytogenetic characteristics had a pronounced weight in this scoring system. Only two patients in this study were classified as good risk and 15 patients as poor-risk. The survival of the intermediate and poor-risk patients was not significantly different $(p=0.29)$, but the numbers are small (Table 1).

GO has been administered as a single dose of $5 \mathrm{mg} / \mathrm{m}^{2}$ on day 7 of the chemotherapy schedule in the current study. It is possible that a more fractioned schedule with a higher total dose of GO might be a more effective strategy which may take advantage of CD33-re-expression that occurs after initial exposure to the GO [31]. The French Alfa-group utilized a GO schedule of $3 \mathrm{mg} / \mathrm{m}^{2} /$ day on days 1,4 , and 7 during induction chemotherapy in patients aged 50-70 years with untreated primary AML. Complete response was $81 \%$ and event-free survival was $40.8 \%$ compared to $17.1 \%$ in the control group ( $p$ value $=0.003)$ [32]. This benefit was also apparent in patients with unfavorable cytogenetic characteristics, but the impact of complex karyotype has not been analyzed separately [32]. In addition, early mortality seems to be reduced if a dose of $3 \mathrm{~g} / \mathrm{m}^{2}$ is used either as a single dose or in a fractionated schedule [27].

\section{Conclusions}

The observed CR/CRi's rate of $43 \%$ led to the conclusion that IAGO is not sufficiently active to warrant further exploration in high-risk MDS and secondary AML evolved from MDS. In addition, patients with poor cytogenetic features had a very poor outcome caused by a complete absence $\mathrm{CR}$ and CRi rate in this phase II study. Alternative schedules with more fractionated schedules of GO may result in better outcome in this population of high-risk MDS or AML evolved from MDS.

Acknowledgments This study was partly supported by an educational grant provided by Wyeth/Pfizer, and by the EORTC Charitable Trust.

Conflict of interest The authors declare that they have no conflict of interest.

Compliance with ethical standards All human studies have been approved by the appropriate ethics committee and have been performed in accordance with the ethical standards laid down in the 1964 Declaration of Helsinki and its later amendments. All procedures followed were in accordance with the ethical standards of the responsible committee on human experimentation (institutional and national) and with the Helsinki Declaration of 1975, as revised in 2008.

Open Access This article is distributed under the terms of the Creative Commons Attribution 4.0 International License (http:// creativecommons.org/licenses/by/4.0/), which permits unrestricted use, distribution, and reproduction in any medium, provided you give appropriate credit to the original author(s) and the source, provide a link to the Creative Commons license, and indicate if changes were made.

\section{References}

1. Stone RM (2009) How I, treat patients with myelodysplastic syndromes. Blood 113(25):6296-6303

2. Oosterveld M, Suciu S, Muus P, Germing U, Delforge M, Belhabri A et al (2015) Specific scoring systems to predict survival of patients with high-risk myelodysplastic syndrome (MDS) and de novo acute myeloid leukemia (AML) after intensive antileukemic treatment based on results of the EORTC-GIMEMA AML-10 and intergroup CRIANT studies. Ann Hematol 94(1):23-34

3. Malcovati L, Hellstrom-Lindberg E, Bowen D, Ades L, Cermak J, Del Canizo C et al (2013) Diagnosis and treatment of primary myelodysplastic syndromes in adults: recommendations from the European LeukemiaNet. Blood 122(17):2943-2964

4. Fenaux P, Morel P, Rose C, Lai JL, Jouet JP, Bauters F (1991) Prognostic factors in adult de novo myelodysplastic syndromes treated by intensive chemotherapy. Br J Haematol 77(4):497-501

5. Hast R, Hellstrom-Lindberg E, Ohm L, Bjorkholm M, Celsing F, Dahl IM et al (2003) No benefit from adding GM-CSF to induction chemotherapy in transforming myelodysplastic syndromes: better outcome in patients with less proliferative disease. Leuk Off J Leuk Soc Am Leuk Res Fund UK 17(9):1827-1833

6. Germing U, Hildebrandt B, Pfeilstocker M, Nosslinger T, Valent P, Fonatsch $C$ et al (2005) Refinement of the international prognostic scoring system (IPSS) by including LDH as an additional prognostic variable to improve risk assessment in patients with primary myelodysplastic syndromes (MDS). Leuk Off J Leuk Soc Am Leuk Res Fund UK 19(12):2223-2231

7. Estey E, Thall P, Beran M, Kantarjian H, Pierce S, Keating M (1997) Effect of diagnosis (refractory anemia with excess blasts, refractory anemia with excess blasts in transformation, or acute myeloid leukemia [AML]) on outcome of AML-type chemotherapy. Blood 90(8):2969-2977

8. de Witte T, Muus P, De PB, Haanen C (1990) Intensive antileukemic treatment of patients younger than 65 years with myelodysplastic syndromes and secondary acute myelogenous leukemia. Cancer 66(5):831-837

9. de Witte T, Suciu S, Peetermans M, Fenaux P, Strijckmans P, Hayat $M$ et al (1995) Intensive chemotherapy for poor prognosis myelodysplasia (MDS) and secondary acute myeloid leukemia (sAML) following MDS of more than 6 months duration. A pilot study by the Leukemia Cooperative Group of the European Organisation for Research and Treatment in Cancer (EORTCLCG). Leuk Off J Leuk Soc Am Leuk Res Fund UK 9(11):18051811

10. Parker JE, Pagliuca A, Mijovic A, Cullis JO, Czepulkowski B, Rassam SM et al (1997) Fludarabine, cytarabine, G-CSF and idarubicin (FLAG-IDA) for the treatment of poor-risk myelodysplastic syndromes and acute myeloid leukaemia. $\mathrm{Br} \mathrm{J}$ Haematol 99(4):939-944

11. Burnett AK, Hills RK, Milligan DW, Goldstone AH, Prentice AG, McMullin MF et al (2010) Attempts to optimize induction and 
consolidation treatment in acute myeloid leukemia: results of the MRC AML12 trial. J Clin Oncol 28(4):586-595

12. Hofmann WK, Heil G, Zander C, Wiebe S, Ottmann OG, Bergmann L et al (2004) Intensive chemotherapy with idarubicin, cytarabine, etoposide, and G-CSF priming in patients with advanced myelodysplastic syndrome and high-risk acute myeloid leukemia. Ann Hematol 83(8):498-503

13. Lepelley P, Soenen V, Preudhomme C, Lai JL, Cosson A, Fenaux P (1994) Expression of the multidrug resistance P-glycoprotein and its relationship to hematological characteristics and response to treatment in myelodysplastic syndromes. Leuk Off J Leuk Soc Am Leuk Res Fund UK 8(6):998-1004

14. Sato H, Gottesman MM, Goldstein LJ, Pastan I, Block AM, Sandberg AA et al (1990) Expression of the multidrug resistance gene in myeloid leukemias. Leuk Res 14(1):11-21

15. Sonneveld P, van Dongen JJ, Hagemeijer A, van Kapel L, Nooter K, Schoester M et al (1993) High expression of the multidrug resistance P-glycoprotein in high-risk myelodysplasia is associated with immature phenotype. Leuk Off J Leuk Soc Am Leuk Res Fund UK 7(7):963-969

16. Leith CP, Kopecky KJ, Godwin J, McConnell T, Slovak ML, Chen IM et al (1997) Acute myeloid leukemia in the elderly: assessment of multidrug resistance (MDR1) and cytogenetics distinguishes biologic subgroups with remarkably distinct responses to standard chemotherapy. A Southwest Oncology Group study. Blood 89(9): 3323-3329

17. de Witte T, Hagemeijer A, Suciu S, Belhabri A, Delforge M, Kobbe $\mathrm{G}$ et al (2010) Value of allogeneic versus autologous stem cell transplantation and chemotherapy in patients with myelodysplastic syndromes and secondary acute myeloid leukemia. Final results of a prospective randomized European Intergroup Trial. Haematologica 95(10):1754-1761

18. Hinman LM, Hamann PR, Wallace R, Menendez AT, Durr FE, Upeslacis J (1993) Preparation and characterization of monoclonal antibody conjugates of the calicheamicins: a novel and potent family of antitumor antibiotics. Cancer Res 53(14):3336-3342

19. Linenberger ML (2005) CD33-directed therapy with gemtuzumab ozogamicin in acute myeloid leukemia: progress in understanding cytotoxicity and potential mechanisms of drug resistance. Leuk Off J Leuk Soc Am Leuk Res Fund UK 19(2):176-182

20. Amadori S, Suciu S, Selleslag D, Stasi R, Alimena G, Baila L et al (2010) Randomized trial of two schedules of low-dose gemtuzumab ozogamicin as induction monotherapy for newly diagnosed acute myeloid leukaemia in older patients not considered candidates for intensive chemotherapy. A phase II study of the EORTC and GIMEMA leukaemia groups (AML-19). Br J Haematol 149(3):376-382

21. Amadori S, Suciu S, Willemze R, Mandelli F, Selleslag D, Stauder $\mathrm{R}$ et al (2004) Sequential administration of gemtuzumab ozogamicin and conventional chemotherapy as first line therapy in elderly patients with acute myeloid leukemia: a phase II study (AML-15) of the EORTC and GIMEMA leukemia groups. Haematologica 89(8):950-956
22. Amadori S, Suciu S, Stasi R, Salih HR, Selleslag D, Muus P et al (2013) Sequential combination of gemtuzumab ozogamicin and standard chemotherapy in older patients with newly diagnosed acute myeloid leukemia: results of a randomized phase III trial by the EORTC and GIMEMA consortium (AML-17). J Clin Oncol Off J Am Soc Clin Oncol 31(35):4424-4430

23. Sievers EL, Larson RA, Stadtmauer EA, Estey E, Lowenberg B, Dombret $\mathrm{H}$ et al (2001) Efficacy and safety of gemtuzumab ozogamicin in patients with CD33-positive acute myeloid leukemia in first relapse. J Clin Oncol Off J Am Soc Clin Oncol 19(13):3244 3254

24. Cheson BD, Greenberg PL, Bennett JM, Lowenberg B, Wijermans PW, Nimer SD et al (2006) Clinical application and proposal for modification of the International Working Group (IWG) response criteria in myelodysplasia. Blood 108(2):419-425

25. Wattel E, Solary E, Hecquet B, Caillot D, Ifrah N, Brion A et al (1998) Quinine improves the results of intensive chemotherapy in myelodysplastic syndromes expressing $\mathrm{P}$ glycoprotein: results of a randomized study. Br J Haematol 102(4):1015-1024

26. Ossenkoppele GJ, Graveland WJ, Sonneveld P, Daenen SM, Biesma DH, Verdonck LF et al (2004) The value of fludarabine in addition to ARA-C and G-CSF in the treatment of patients with high-risk myelodysplastic syndromes and AML in elderly patients. Blood 103(8):2908-2913

27. Hills RK, Castaigne S, Appelbaum FR, Delaunay J, Petersdorf S, Othus $\mathrm{M}$ et al (2014) Addition of gemtuzumab ozogamicin to induction chemotherapy in adult patients with acute myeloid leukaemia: a meta-analysis of individual patient data from randomised controlled trials. Lancet Oncol 15(9):986-996

28. Burnett AK, Hills RK, Milligan D, Kjeldsen L, Kell J, Russell NH et al (2011) Identification of patients with acute myeloblastic leukemia who benefit from the addition of gemtuzumab ozogamicin: results of the MRC AML15 trial. J Clin Oncol 29(4):369-377

29. Burnett AK, Russell NH, Hills RK, Kell J, Freeman S, Kjeldsen L et al (2012) Addition of gemtuzumab ozogamicin to induction chemotherapy improves survival in older patients with acute myeloid leukemia. J Clin Oncol Off J Am Soc Clin Oncol 30(32):3924 3931

30. de Witte T, Suciu S, Verhoef G, Labar B, Archimbaud E, Aul C et al (2001) Intensive chemotherapy followed by allogeneic or autologous stem cell transplantation for patients with myelodysplastic syndromes (MDSs) and acute myeloid leukemia following MDS. Blood 98(8):2326-2331

31. van Der Velden VH, te Marvelde JG, Hoogeveen PG, Bernstein ID, Houtsmuller AB, Berger MS et al (2001) Targeting of the CD33calicheamicin immunoconjugate Mylotarg (CMA-676) in acute myeloid leukemia: in vivo and in vitro saturation and internalization by leukemic and normal myeloid cells. Blood 97(10):3197-3204

32. Castaigne S, Pautas C, Terre C, Raffoux E, Bordessoule D, Bastie JN et al (2012) Effect of gemtuzumab ozogamicin on survival of adult patients with de-novo acute myeloid leukaemia (ALFA0701): a randomised, open-label, phase 3 study. Lancet 379(9825):1508-1516 